\title{
Comparative Study between the Effect of Skeletonized and Pedicled Internal Thoracic Artery on Sternum Healing in Patient Undergoing CABG
}

\author{
Yousry A. Shaheen ${ }^{\text {a }}$, Ibrahim R. Kasab ${ }^{\text {a }}$, Mostafa Galal ${ }^{\text {b }}$
}

\begin{abstract}
a Department of Cardiothoracic Surgery, Benha faculty of medicine, Benha University, Egypt. ${ }^{b}$ Department of cardiothoracic surgery, Elzaitoun specialized hospital, Cairo, Egypt.

Correspondence to: Mostafa Galal, Department of cardiothoracic surgery, Elzaitoun specialized hospital, Cairo, Egypt.
\end{abstract}

Email:

most.galal@gmail.com

Received: 8 January 2020

Accepted: 6 April 2020

\section{Abstract:}

Background: Myocardial revascularization is performed preferentially with internal thoracic artery grafts. This study aimed to investigate sternal healing over time and the incidence of poor sternal healing and wound infection in patients undergoing coronary artery bypass graft $(\mathrm{CABG})$ surgery using skeletonized internal thoracic artery. Objectives: (1) comparison of two surgical take-down techniques (skeletonizing vs. standard pedicle harvestation); (2) assessment of sternal healing and wound infection. Method: This study enrolled 50 patients (divided in two groups) who underwent isolated $\mathrm{CABG}$ using skeletonized internal thoracic artery (25 patients) and pedicled internal thoracic artery (25 patients) from December 2017 to August 2019. Postoperative assessment was performed on all patients for three months after surgery. In all patients, chest X-ray study was performed after surgery to assess sternal healing. Medical records were also retrospectively reviewed to identify perioperative variables associated with poor early sternal healing. Results: (1) After coronary grafting, assessment of sternum showed significant differences in healing between the two groups. (2) Diabetes mellitus as an independent predictor for superficial wound infection. (3) Pedicled procedure is an important factor associated with superficial wound infection in post-operative period. (4) Skeletonized procedure prolong the operation. (5) Pedicled procedure prolong the ICU and hospital stay.Conclusion: (1) Skeletonization of internal thoracic artery conduits lowers the risk of superficial and deep sternal wound infection in patients undergoing CABG with internal thoracic artery grafting. (2) Skeletonization of internal thoracic artery conduits prolong the operation time and require more surgical skills. (3) Skeletonization of ITA has better outcome. 


\section{Introduction:}

The Internal thoracic artery (ITA) plays a vital role in coronary artery bypass grafting. The left ITA is the gold standard conduit in grafting the left anterior descending coronary artery. During CABG the internal Thoracic artery (ITA) may be harvested either as a pedicle together with concomitant veins, lymphatics, sympathetic plexus and internal Thoracic fascia or skeletonized free of all surrounding tissues. $(1,2)$

There is insufficient evidence to prompt the surgeon to harvest the ITA in a skeletonized fashion. Randomized studies comparing the skeletonized to the Pedicled ITA are few, and most of the current knowledge on this subject comes from observational studies that offer insufficient evidence for or against skeletonization of the ITA. ${ }^{(3)}$

Slow or poor healing of the sternum is one of the potential problems encountered after $\mathrm{CABG}$ and is therefore one of the potential problems associated with ITA harvesting. Slow healing prolongs the hospital stay, increases health care costs considerably, and delays the return to work or social activities. Poor healing of the sternum often leads to deep sternal wound infection, which is serious complication. ${ }^{(4)}$

\section{Patients:}

This randomized study included 50 patients diagnosed as IHD, and needed surgical myocardial revascularization $(\mathrm{CABG})$ in Cardiothoracic Surgery Department in Benha Faculty of Medicine and El-Zyton Specialized hospital in the duration from December 2017 to August 2019 for patients who fulfilled the following criteria: (Patients with IHD indicated for Isolated elective $\mathrm{CABG}$ within that mention period, Multi-vessels disease, Age 30 to 70 years, Males and female, HbA1c $<7$ and Body mass index <30).

Patients were submitted for either group in randomized way and performed by many surgeons for each group as follow: Group A: (25 patients): CABG using pedicled left internal mammary artery. Group B: (25 patients): $\mathrm{CABG}$ using skeletonized left internal mammary artery.

\section{Methods:}

Preoperative stage of preparation / assessment included standard steps in all patients which started by careful and thorough history taking and clinical examination taking into consideration the patient's following items: (age, sex, weight, BSA, diabetes, hypertension, hyperlipidemia, smoking left main lesion, 
previous stent, left ventricle ejection fraction, Number of vessels and routine investigation of $\mathrm{CABG}$ patients.

Surgical technique pedicled ITA: An incision using the electrocautery at low energy setting is made in the parietal pleura and muscle on the medial side of the ITA, several millimeters from the accompanying internal thoracic vein. The incision is extended along most of the length of the vessel down to the sixth intercostal space. A parallel incision lateral to the ITA and the accompanying lateral internal thoracic vein (if present) is made.

The correct plane was entered by reflecting the pleura and transversus thoracic muscle behind the sternum. Dissection is begun in the sixth intercostal space, where there are usually no branches. Using the tip of the electrocautery blade without current, the pedicle is freed from the sixth costal cartilage. With the pedicle gently retracted downward and with gentle blunt dissection, the intercostal arteries are identified and either occluded with small metal clips and divided, or simply divided with the electrocautery. The ITA should not be grasped directly with instruments. After completing its proximal dissection, heparin is administered before division is done at 67th space. With the proximal end controlled using a small bulldog clamp and the distal end ligated or clipped.

Skeletonization of ITA: A median sternotomy was performed. After dissecting the reflection of the mediastinal pleura from the endothoracic fascia without opening of pleura, the ITA and both satellite veins were visualized. The endothoracic fascia was longitudinally incised just medial to one of the satellite veins using the electrocautery at low energy setting. The space between the medial satellite vein and the ITA was carefully dissected. Only the ITA was targeted for dissection. The fatty tissue around the ITA was foamed and easily removed by lightly sweeping it away, moving the electrocautery at low power along the length of the vessel to expose the adventitia of the ITA. Importantly, the contact time of the electrocautery at low power was set at 0.1 to 0.2 seconds and the scalpel was moved lightly and quickly along several centimeters of the vessel as main trunk ("quick touch" method). In this way, the main trunk was skeletonized safely and quickly. In contrast, treatment of the branches is a slower process. After exposing a branch, in almost all patients, clip was needed for ITA branch closure. Branches should never be separated by pulling the hook inside the tip of the blade. Venous branches rarely cross lateral to medial 
anterior to the ITA, and when they do, it usually is in the vicinity of the third or fourth interspace. These veins were doubly clipped and cut or ablated by coagulation. The ITA was then separated from the fascia, with completion of skeletonization.

After pericardiotomy, routine aortic and right atrial cannulation was done using a common single atrial venous cannula. A double outlet cardioplegia cannula was inserted in the aortic root in all cases. Cardiopulmonary bypass was carried out at normothermia in all cases. Intraoperative myocardial protection was achieved using antegrade intermittent warm blood cardioplegia starting with the initial dose $300 \mathrm{ml} /$ minute for 3 minutes containing 15 MEq of Potassium Chloride. All distal anastomoses were constructed during one period of aortic cross clamping. Additional doses of cardioplegia were given after completion of each anastomotic point (200 $\mathrm{ml} / \mathrm{minute}$ for 2 minutes containing $6 \mathrm{mEq}$ of KCL. Venting of the heart was carried out through the same cardioplegia cannula double-line via the aortic root.

Performing the distal anastomoses was usually performed in the same sequence for all cases of both groups. Initially, the right coronary artery was started with followed by the posterior RCA branches (crux or
PDA), then the marginal branches of the circumflex artery, and the diagonal branches of the LAD. The integrity of the grafts was carefully checked for flow and any possible leakage from the suture line before the LITA is anastomosed to the LAD vessel as a final step in all patients. The distal anastomoses in all cases were done in an end-to-side fashion. Running polypropylene $7 / 0$ sutures were used for the LITA. After all distal anastomoses were finished, and before removal of aortic cross clamping, all patients were given a hot shot of pure warm oxygenated blood at a rate of $300 \mathrm{mls} / \mathrm{minute}$ for 3 minutes (without $\mathrm{KCl}$ ), via the cardioplegia line. Two grams of magnesium sulphate was given via the same line upon removal of the aortic cross clamp. The proximal anastomoses in all cases were done using Running polypropylene $6 / 0$ sutures.

Sternum closure: The sternum is closed with stainless steel wire using figure of eight technique. Muscles is closed with vicryl 2 round suture and skin is closed with vicryl $2 / 0$ cutting suture.

A record was made of the following: (Skeletonized or Pedicled Internal Thoracic Artery, Cardiopulmonary Bypass Time, Cross clamp time, Number of vessels) 
The results of CABG will be assessed using the following: (reopening for bleeding, duration of mechanical ventilation, duration of ICU stay, duration of hospital stay, superficial sternal wound infection, deep sternal wound infection, sternal dehiscence, rewire of sternum and chest X-Ray.

Data were expressed as mean $+(\mathrm{SD})$ or compare the means of values in the 2 patients' groups. The distribution of qualitative variables across groups was analyzed by Chi- square test or Fischer's exact test as appropriate. All comparisons were bilateral and a $\mathrm{P}$ value $<0.05$ was the limit of statistical significance. Analysis was performed by SPSS statistical software version 23 for windows.

\section{Results:}

The patient demographics (age, height, weight, BSA and LVEF) are showing no statistical differences among the two groups as expressed in table 1.

There are large number of male patients than females and many hypertensive patients in both groups. Also it shows more diabetic, hyperlipedemic and smoker patients among skeletonized group than pedicled group as shown in table 2 .

Table 1: patients' demographics.

\begin{tabular}{llll}
\hline Variables & Pedicled $=\mathbf{2 5}$ patients & Skeletonized $=\mathbf{2 5}$ patients & P- value \\
\hline & Mean \pm SD & Mean \pm SD & \\
Age (Years) & $60.20 \pm 1.21$ & $59.92 \pm 1.29$ & 0.96 \\
Height $(\mathbf{m})$ & $1.68 \pm .023$ & $1.67 \pm 0.022$ & 0.84 \\
Weight $($ Kg) & $83.84 \pm 2.30$ & $82.36 \pm 2.28$ & 0.86 \\
BMI & $29.51 \pm .311$ & $29.34 \pm 0.38$ & 0.61 \\
LVEF \% & $43.83 \pm 10.05$ & $40.88 \pm 7.75$ & 0.74 \\
\hline
\end{tabular}

Table 2: patients' risk factors.

\begin{tabular}{llcccc}
\hline & Variables & \multicolumn{2}{c}{ Pedicled=25 patients } & \multicolumn{2}{c}{ Skeletonized=25 patients } \\
& & Number & Percent \% & Number & Percent \% \\
\hline Sex. & male & 15 & $60 \%$ & 16 & $65 \%$ \\
DM. & female & 10 & $40 \%$ & 9 & $45 \%$ \\
& Yes & 11 & $44 \%$ & 17 & $68 \%$ \\
HTN. & No & 14 & $56 \%$ & 8 & $32 \%$ \\
\multirow{3}{*}{ Hyperlipidemia. } & Yes & 23 & $92 \%$ & 21 & $84 \%$ \\
\multirow{3}{*}{ Smoking } & No & 2 & $8 \%$ & 4 & $16 \%$ \\
& Yes & 13 & $52 \%$ & 16 & $64 \%$ \\
& No & 12 & $48 \%$ & 9 & $36 \%$ \\
& Yes & 12 & $48 \%$ & 11 & $45 \%$ \\
& No & 13 & $52 \%$ & 14 & $56 \%$ \\
\hline
\end{tabular}


There is no significant difference between two groups regarding number of vessels, skeletonized group versus pedicled group $(2.72 \pm 0.89$ versus $2.68 \pm 0.90)$, moreover the bypass time showed no significant difference between skeletonized group and pedicled group, it was $(95.92 \pm 25.629$ and $96.52 \pm 24.4)$ respectively. However the mean time of LITA harvesting was more in skeletonized group than the pedicled group $(55.69 \pm 8.80$ versus $44.28 \pm 6.95)$ but with no statistical significant difference as shown in table 3.

There is no significant difference in P-value between two groups regarding postoperative drainage, duration of ventilation and ICU stay while, there is significant difference between two groups in mean hospital stay (skeletonized group versus pedicled group) (11.6 \pm 3.5 versus $7.88 \pm$ 1.90) (P-value 0.04) as demonstrated in table 4.

The sum of all patients in the two groups was 50 patients, 4 of them were reopened for bleeding, 8 of them developed superficial wound infection, 3 of them developed deep sternal wound infection and one patient went for rewiring of sternum. The skeletonized group showed less complications rather than pedicled group regarding reopening for bleeding (1case versus 3 cases), superficial sternal wound infection( 2 cases versus 6 cases), deep sternal wound infection (1case versus 2 cases) and rewire of sternum ( 0 cases versus 1 case) as demonstrated in table 5 .

Table 3: Group comparison of operative data.

\begin{tabular}{lccc}
\hline \multicolumn{1}{c}{ Variables } & Pedicled $=\mathbf{2 5}$ patients & Skeletonized $=$ 25 patients & P- value \\
& Mean \pm SD & Mean \pm SD & \\
\hline Time of harvesting $(\mathbf{m i n})$ & $44.28 \pm 6.95$ & $55.69 \pm 8.80$ & 0.09 \\
No. of vessels & $2.68 \pm 0.90$ & $2.72 \pm 0.89$ & 0.84 \\
Bypass time (min) & $96.52 \pm 24.4$ & $95.92 \pm 25.629$ & 0.81 \\
\hline
\end{tabular}

Table (4): Post-operative data.

\begin{tabular}{lccc}
\hline \multicolumn{1}{c}{ Variables } & $\begin{array}{c}\text { Pedicled }=\mathbf{2 5} \text { patients } \\
\text { Mean } \pm \text { SD }\end{array}$ & $\begin{array}{c}\text { Skeletonized }=\mathbf{2 5} \text { patients } \\
\text { Mean } \pm \text { SD }\end{array}$ & P- value \\
\hline $\begin{array}{lcc}\text { Post-Operative drainage (ml / 24 } \\
\text { hours) }\end{array}$ & $610 \pm 132.28$ & $442.36 \pm 166.06$ & 0.7 \\
Duration of ventilation (hours) & $13 \pm 3.01$ & & \\
ICU stay (days) & $2.88 \pm 0.781$ & $9.36 \pm 3.174$ & 0.83 \\
Hospital stay (days) & $11.6 \pm 3.5$ & $2.28 \pm 0.678$ & 0.6 \\
\hline
\end{tabular}


Table 5: Post-operative complications

\begin{tabular}{llcccc}
\hline Variables & \multicolumn{2}{c}{ Pedicled=25 patients } & \multicolumn{3}{c}{ Skeletonized= 25 patients } \\
& & Number & Percent \% & Number & Percent \% \\
\hline Reopen for Bleeding & Yes & 3 & $12 \%$ & 1 & $4 \%$ \\
& No & 22 & $88 \%$ & 24 & $96 \%$ \\
superficial sternal wound infection & Yes & 6 & $24 \%$ & 2 & $8 \%$ \\
& No & 19 & $76 \%$ & 23 & $92 \%$ \\
deep sternal wound infection & Yes & 2 & $8 \%$ & 1 & $4 \%$ \\
& No & 23 & $92 \%$ & 24 & $96 \%$ \\
Rewire of sternum & Yes & 1 & $4 \%$ & 0 & $0 \%$ \\
& No & 24 & $96 \%$ & 25 & $100 \%$ \\
\hline
\end{tabular}

\section{Discussion:}

Arterial grafting during coronary bypass surgery is routinely performed as a result of the success of left ITA grafting of the left anterior descending artery. Compared with saphenous vein bypass grafting, patients who receive an ITA to the left anterior descending artery survive longer and experience less hospitalization for cardiac events, lower rates of cardiac reoperation, and less frequent myocardial infarction. ${ }^{(5)}$

The ITA may be harvested as a pedicle or as a skeletonized graft, with evidence suggesting that pedicled ITA may affect sternal healing to a greater extent. Therefore, understanding the impact of ITA harvesting techniques on sternal healing is important for cardiac surgeons to tailor the optimal conduit harvesting strategy to each patient undergoing $\mathrm{CABG}{ }^{\left({ }^{(6)}\right.}$

We think that several reasons exist for delayed sternal Healing. Carmelo A. Milano and his colleagues reported that obesity and old age are major factors which affect and decrease sternal healing. ${ }^{(7)}$ Nagachinta et al identified obesity as the strongest predictor, with $14 \%$ of the obese patients developing mediastinitis. (8) Loop et al identified obesity as the second most important independent predictor of mediastinitis, defining obesity as only a $20 \%$ increase in height-adjusted weight. ${ }^{(9)}$ In our study, the patients selected in the two groups were approximately the same regard age, weight, height and BMI with insignificant $\mathrm{P}$ value. Another factor that affect the sternal healing is diabetes mellitus. Our study shows that 8 
out $50(16 \%)$ of all patients developed superficial wound infection. 6 out of 8 $(75 \%)$ were diabetic and 2 out of $6(34 \%)$ of those diabetic patient who developed superficial wound infection were in skeletonized group while 4 out of $6(66 \%)$ were in pedicled group. These results point to diabetes mellitus as an independent predictor for superficial wound infection, Moreover these results show that the incidence of superficial wound infection was more in pedicled group compared to skeletonized group, which reveal that pedicled procedure is an important factor associated with superficial wound infection in post-operative period.

Most studies assessing sternal healing have compared the effect of surgical technique on harvesting ITA either skeletonized or pedicled were limited by comparing bilateral skeletonized ITA versus bilateral pedicled ITA, or bilateral skeletonized ITA versus single skeletonized ITA. In the present study the graft arrangement was strictly controlled with only the LITA to LAD and saphenous vein graft to other coronary arteries to minimize other factors affecting the healing of the sternum, so the simple role of skeletonized technique in single ITA was well evaluated.

In our study, Time of bypass was 40 approximately the same in the two groups with $(\mathrm{p}$ value $=0.8)$. However harvesting time showed near significant difference in favor to pedicled group as it was (44.28 \pm $6.95)$ for pedicled group and $(55.69 \pm 8.80)$ for skeletonized group with $(\mathrm{p}=0.09)$. These results show that pedicled technique has a minor priority against skeletonized technique as it take less harvesting time and hence less operative time. Carmelo and his colleagues found that Prolonged CPB time and operative time were significant independent predictors of decreasing the sternum healing and increasing the incidence of mediastinitis. ${ }^{(7)}$ Comparing to our results we can see that prolonged harvesting time which indeed prolong the total operative time may increase the incidence of superficial and deep sternal wound infection and hence the skeletonized technique may increase the incidence of superficial and deep sternal wound infection.

Sergio Barsanti and his colleagues in there study reported that prolonged intensive care unit stay increase the risk of wound infection. ${ }^{(10)}$ In our study, there was no significant difference between the two groups regard ICU time post-operative. However there was a significant difference in Hospital stay in favor to skeletonized 
group as it was $(11.6 \pm 3.5)$ in pedicled group and $(7.88 \pm 1.90)$ in skeletonized group with $(\mathrm{p}=0.04)$. This revel that pedicled group was more liable to develop post-operative infected wound more than skeletonized group.

The need for early re-exploration for bleeding is defined as the need for resternotomy within 24 hours of the operation. This is considered an independent risk for superficial and deep sternal wound infection according to Michael et al ${ }^{(11)}$. In our study the difference between pedicled group and skeletonized group was insignificant regard amount of bleeding in the first 24 hours post-operative with $(\mathrm{P}=0.7)$, however the number of cases we re-explored for bleeding was more in pedicled group (3 cases - 12\%) than in skeletonized group (1 case $-4 \%$ ). It means that pedicled group was more liable for early re-exploration which may be due to large surgical raw area during LITA harvesting as pedicled compared to a small surgical raw area during LITA harvesting as skeletonized. This indicates that skeletonization of LITA may decrease the risk of early re-exploration for bleeding and hence decreasing the risk of superficial and deep sternal wound infection.

In our study, there were 8 patients who developed wound infection, only one patient underwent sternum rewiring. This patient was a male 57 year's old, smoker, hypertensive and diabetic with BMI 30. The patient went for $\mathrm{CABG}$ and ITA was pedicled. The operation time was long (110 min) and patient was ventilated for 21 hours. The patient was re-explored for bleeding after drain was $800 \mathrm{cc}$ in the first 6 hours. ICU stay was 4 days and hospital stay was 19 days. The patient developed superficial wound infection then deep wound infection and lately developed sternum dehiscence. The patient went for sternal rewire.

This case represented many risk factors that predispose to wound infection such as diabetes, however the patient was not morbid obese with BMI 30. The main risk factor was the pedicled technique of harvesting LITA as it caused large surgical raw area and the patient's blood drainage was large amount (800cc in the first 6 hours) which was the main cause for resternotomy to explore the cause of bleeding. This course could be avoided if The LITA was harvested as a skeletonized with its small surgical raw area which cause less bleeding indeed. The surgical re-sternotomy caused more surgical damage to the tissue beside the need to close the sternum with a 
new wire and the tissues with a new sutures, this more manipulation caused more tissue damage which decreased the healing process and increased the risk for wound infection.

The wound developed superficial wound infection and developed to deep wound infection and sternal dehiscence. The patient went for a sternum rewire to stabilize the sternum and stayed at hospital for 19 days.

The aim of our study is to compare the effect of Pedicled and skeletonized LITA and determine which technique is better regard the sternum healing. There are many points in favor of each technique which make it difficult to favor one. The Pedicled LITA is the standard technique which take short harvesting time and less training time, however the skeletonized LITA takes longer harvesting time and requires more surgical training and experience. The skeletonized LITA cause less bleeding, less ICU stay, less hospital stay and indeed less wound complications.

\section{References:}

(1) Robert F,Thomas A, Kamellia R, et al. Coronary Artery Bypass Graft Surgery Using the Radial Artery, Right Internal Thoracic Artery, or Saphenous Vein as the Second Conduit. The Annals of Thoracic Surgery 104 (2017) 553-559.

\section{Conclusion:}

The skeletonization technique is recommended in patients with risk factor that may affect sternal healing like obesity and diabetes mellitus, as our study showed that it decreases the risk of post-operative wound infection rather than the pedicled technique. Another reason to recommend the skeletonized technique is that it maintains a closed pleura which preserve the respiratory function and decrease the ventilation period. This study reveals the impact of pedicled technique on increasing the risk of prolonged ventilation period, prolonged hospital stay and decreased sternal healing. However the skeletonized technique is not the standard technique as it take more time on operation and require advanced surgical training to harvest the ITA perfectly without causing harm to it.

(2)

Carlos D. Pedicled or Skeletonized? A Review of the Internal Thoracic Artery Graft. Texas Heart Institute,J 2003;30: 170-5.

(3) Marek A, Krzysztof S, Marcin M, et al. Skeletonization of internal thoracic artery affects its innervation and reactivity. European Journal of Cardiothoracic Surgery 28 (2005) 551-557. 
Benha medical journal vol. 37, Special issue (surgery), 2020

(4) Shukri F. To Skeletonize the Internal Thoracic Artery or Not? Is That the Question? Circulation. 2006; 114:754-756.

(5) Peterson M, Borger M, Rao V. Skeletonization of bilateral internal thoracic artery grafts lowers the risk of sternal infection in patients with diabetes. $\mathbf{J}$ Thorac Cardiovasc Surg 2003;126:1314-9.

(6) Kevin C, Syed R and David T. A Review of Differing Techniques of Mammary Artery Harvesting on Sternal Perfusion: Time for a Randomized Study. Ann Thorac Surg 2015;100:1942-53.

(7) Carmelo A, Karen K, Nancy A, Daniel J, and Robert H. Mediastinitis After Coronary Artery Bypass Graft Surgery. Circulation. 1995;92:2245-2251.
(8) Nagachinta T, Stephens M, Reitz B, Polk BF. Risk factors for surgical wound infection following cardiac surgery. J Infect Dis.1987; 156:967-973..

(9) Loop FD, Lylte BW , cosgrove DM, Mahfood S, McHenry MC, et al. sternal wound complications after isolated coronary artery bybass grafting:early and late mortality, morbidity, and cost of care. The Annals of Thoracic Surgery. 1990;49: 179-187.

(10) Sergio B, Cely A, Valeria T. Risk factors for mediastinitis after cardiac surgery. The Annals of Thoracic Surgery. 2004;77: 676-683.

(11) Michael J, Lawrence C, Mary E, Mackey BA, JamesCox, Michael R. Reexploration for bleeding is a risk factor for adverse outcomes after cardiac operations. The Journal of Thoracic and Cardiovascular Surgery. 1996;111: 1037-1046.

To cite this article: Yousry A. Shaheen, Ibrahim R. Kasab , Mostafa Galal Comparative Study Between the effect of Skeletonized and Pedicled Internal Thoracic Artery on sternum healing in Patient Undergoing CABG. BMFJ 2020; 37 (surgical issue): 33-43, DOI: 10.21608/bmfj.2020.22151.1203 